\title{
DYNAMICS OF HEAT TRANSFER IN THE MELT POOL AT NUCLEAR SEVERE ACCIDENT CONDITIONS
}

\author{
Andrej Horvat , Ivo Kljenak \\ "Jožef Stefan" Institute \\ Reactor Engineering Division \\ Jamova 39, 1001 Ljubljana, Slovenia \\ E-mail: andrej.horvat@ijs.si \\ Jure Marn \\ Faculty of Machanical Engineering \\ University of Maribor \\ Smetanova 17, 2000 Maribor, Slovenia
}

\section{ABSTRACT}

Prediction of thermal loads on nuclear reactor vessel lower plenum after core melting and relocation during a severe accident requires knowledge about the core melt behavior, especially the circulation pattern. To analyze the heat transfer dynamics on the lower plenum walls, two-dimensional numerical simulations of a fluid flow with internal heat generation were performed for Rayleigh numbers $10^{6}, 10^{7}, 10^{8}, 10^{9}, 10^{11}$ and $10^{13}$ at Prandtl number 0.8. For subgrid motion modeling, a Large-Eddy Simulation Smagorinsky model was implemented.

The minimum, time-average and maximum Nusselt numbers on the boundaries were calculated. The dynamics of fluid structures were analyzed to reveal the instability mechanisms and transition to turbulence. Results disclose Rayleigh-Taylor instabilities as a dominant mechanism for turbulence appearance, which occurs when the Rayleigh number is increased over $10^{8}$. The structure dependence of fluid motion at high Rayleigh numbers makes the time-average of heat transfer hard to assess. The time-average values should be supplemented with probability distributions of related variables.

\section{NOMENCLATURE}

$C_{s} \quad$ Smagorinsky constant

$c_{p} \quad$ specific heat

g gravity

I volumetric heat generation

$L \quad$ length of simulation domain

$\mathrm{Nu} \quad$ Nusselt number, $\left(\partial T / \partial x_{i}\right) L /\left(T_{w}-T_{\text {vol.aver }}\right)$

$p \quad$ pressure

Pr Prandtl number $v / v$

Ra Rayleigh number, $\left(|\vec{g}| \beta I L^{5}\right) /(\nu \lambda v)$

$\underline{S} \quad$ deformation velocity tensor

$T$ temperature

$t \quad$ time

$v \quad$ velocity

$\beta \quad$ temperature dilatation

$\lambda \quad$ thermal conductivity

$\rho \quad$ mass density

$\varphi \quad$ mesh generation parameter

$v \quad$ kinematic viscosity

$v \quad$ thermal diffusivity

$\tau \quad$ stress tensor

\begin{tabular}{ll}
\multicolumn{2}{l}{ Subscripts/Superscripts } \\
\hline aver & average \\
$i, j$ & indices \\
$i t$ & iteration counter \\
$n$ & number of grid points \\
sgd & subgrid or turbulent variable \\
$w$ & wall \\
$x$ & horizontal direction \\
$y$ & vertical direction
\end{tabular}

\section{INTRODUCTION}

In a light water reactor, inadequate or prolonged absence of nuclear reactor core cooling may cause core melting to occur. The reactor core melt relocates, flowing downward towards the reactor vessel lower plenum where it accumulates. Heat is further generated in the pool due to fission product decay. Because of high temperature melt, the integrity of the lower plenum could be threatened unless sufficient outside cooling exists. An extensive review of experimental and theoretical results may be found, for example, in the work of Nourgaliev et al. (1997).

In the past few years, the issue of lower plenum coolability during the described severe accident scenario has received much attention. According to the state-of-the-art knowledge about this issue, the reactor vessel integrity would be 
maintained. However, we were not successful in finding either a full-scale experiment or an adequate numerical simulation, which would prove the lower plenum integrity under severe accident conditions.

Prediction of thermal loads on lower plenum walls requires knowledge about the fluid behavior, especially about the circulation pattern, which is, due to high temperature gradients, mainly governed by buoyancy forces in the melt pool. For these reasons the present work is focused on natural convection in the core melt.

Previous experimental and numerical works from various authors often presented only the mean values of Nusselt number at the lower plenum boundaries without describing the uncertainties of obtained results (e.g. Asfia and Dhir, 1996). Such information about thermal load could be misleading. Namely, at higher Rayleigh numbers $\left(\mathrm{Ra}>10^{8}\right)$, the fluid circulation patterns become unsteady, which leads first to local and then to global turbulent motion, so that mean values of Nusselt number do not coincide with most probable values. This suggests that single-value calculations of heat transfer related values in the lower plenum should be supplemented at least with extremal (minimum and maximum) values.

In the present paper, efforts will be presented to identify the mechanisms, which govern the non-linear behavior of the buoyancy flow and thus make the heat transfer loadings on the walls difficult to assess.

\section{MATHEMATICAL MODEL}

The basic assumption in the present work was to consider the melt as an incompressible fluid with internal heat generation. As the boundaries are isothermal, the internal heat generation is the prime mover for natural convection. Following this assumption, the melt behavior is described with mass, momentum and energy equations:

$$
\begin{aligned}
\nabla \cdot \vec{v} & =0 \\
\frac{\partial \vec{v}}{\partial t}+\nabla \cdot(\vec{v} \otimes \vec{v}) & =-\frac{1}{\rho} \nabla p+\nabla \cdot \underline{\tau}-\beta \Delta T \vec{g} \\
\frac{\partial T}{\partial t}+\nabla \cdot(\vec{v} T) & =\frac{\lambda}{c_{p} \rho} \nabla^{2} T+\frac{I}{c_{p} \rho}
\end{aligned}
$$

To reduce the number of free parameters in the calculations and to simplify the comparison of results, eqs. (1), (2) and (3) were transformed into dimensionless form using Boussinesq' $s$ approximation to include buoyancy forces:

$$
\nabla \cdot \vec{v}=0
$$

$$
\frac{\partial \vec{v}}{\partial t}+\nabla \cdot(\vec{v} \otimes \vec{v})=-\nabla p+\operatorname{Pr}(\nabla \cdot \underline{\tau})-\operatorname{Ra} \operatorname{Pr} T \frac{\vec{g}}{|\vec{g}|}
$$

$$
\frac{\partial T}{\partial t}+\nabla \cdot(\vec{v} T)=\nabla^{2} T+1
$$

A complete description of dimensionless variables may be found in Decker (1996) and in Horvat (1998).

\section{Turbulence modeling}

At high Rayleigh numbers $\left(\mathrm{Ra}>10^{8}\right)$ time aperiodic behavior occurs. When the Rayleigh number is further increased $\left(\mathrm{Ra}>10^{9}\right)$ local turbulent motion appears, reducing the local scale of fluid motion.

To properly take into account the subgrid motion of the fluid, a Large-Eddy Simulation (LES) Smagorinsky model was implemented, with a modification to capture also the buoyancy forces due to temperature gradients (as proposed by Eidson, 1985). After applying the Large-Eddy Simulation concept of spatial filtering, eqs. (4), (5) and (6) are written as:

$$
\begin{gathered}
\nabla \cdot \overline{\vec{v}}=0 \\
\frac{\partial \overline{\vec{v}}}{\partial t}+\nabla \cdot(\overline{\vec{v}} \otimes \overline{\vec{v}})= \\
=-\nabla \bar{p}+\operatorname{Pr}\left(\nabla^{2} \overline{\vec{v}}\right)-\operatorname{RaPr} \overline{\frac{g}{\mid \vec{g}} \mid}+\nabla \cdot\left(v_{s g d} 2 \bar{S}\right), \\
\frac{\partial \bar{T}}{\partial t}+\nabla \cdot(\overline{\vec{v}} \bar{T})=\nabla^{2} \bar{T}+1+\nabla \cdot\left(v_{s g d} \nabla \bar{T}\right)
\end{gathered}
$$

where the overbar indicates filtered values. The non-linear convection term of subgrid velocity in the momentum equation acts as a stress term with artificial subgrid viscosity, which is defined according to Eidson (1985) as:

$$
v_{s g d}=\left(C_{s} \Delta x\right)^{2}\left(2 \underline{S}: \underline{S}+\frac{\operatorname{Ra} \operatorname{Pr}}{\operatorname{Pr}_{s g d}}\left(\nabla \bar{T} \cdot \frac{\vec{g}}{|\vec{g}|}\right)\right)^{1 / 2} .
$$

The first term in eq. (10) represents stress forces while the second term represents buoyancy. Similarly, the non-linear convection term of subgrid velocity and temperature in the energy equation can be replaced with diffusive flux using the subgrid thermal diffusity defined as a linear function of subgrid viscosity:

$$
v_{s g d}=\frac{v_{s g d}}{\operatorname{Pr}_{s g d}}
$$

The presented Smagorinsky model is too dissipative in the vicinity of the walls. To reduce the near wall dissipation, the van Driest dumping function was multiplied by the subgrid viscosity. 


\section{Geometrical considerations}

Although experimental and numerical results for semicircular and elliptical cavities are already available (Theofanous et al., 1997), a square cavity was used to simplify calculations at high Rayleigh numbers. The comparison of experimental results from natural convection cases in rectangular and spherical cavities reveals the similarity of heat transfer processes in both geometries. Moreover, it is safe to assume that the maximum Nusselt number is similar for rectangular and spherical cavities of similar dimensions. The maximum Nusselt number occurs in the upper corners of the cavities. Larger discrepancies between heat transfer in rectangular and spherical cavities occur in the lower parts (Asfia and Dhir, 1996; Nourgaliev et al., 1997).

Experimental and numerical results presented by Dinh and Nourgaliev (1997) and Nourgaliev et al. (1997) suggest that the fluid pattern is basically two-dimensional. A two-dimensional model has thus been selected for the simulation of the considered phenomena.

\section{Initial and boundary conditions}

At the simulation beginning, the fluid was considered at rest with an isothermal temperature field.

For the momentum equation, no-slip boundary conditions at all boundaries of the rectangular cavity were prescribed. To represent the solidification and melting processes at the walls of the lower plenum, identical isothermal boundary conditions at all boundaries were prescribed for the energy equation.

\section{NUMERICAL METHODS}

The transport eqs. (7), (8) and (9) were discretized according to a finite volume method using $128 \times 128$ numerical cells. A staggered arrangement for grid points was applied.

The high Rayleigh numbers and modest number of grid points used in the presented calculations required local mesh refinement. This was necessary for accurate simulation of the viscous sublayer behavior at the boundaries of the simulation domain. In the $x$-direction, the numerical mesh was refined according to eq. (12):

$$
\Delta x_{i}=\frac{L_{x}}{n_{x}-1}\left(\varphi+(1-\varphi) \operatorname{Sin}^{2}\left(\pi \frac{i}{n_{x}}\right)\right)
$$

whereas in the $y$-direction, it was refined according to eq. (13):

$$
\Delta y_{j}=\frac{L_{y}}{n_{y}-1}\left(\varphi+(1-\varphi) \operatorname{Sin}^{2}\left(\pi \frac{j}{n_{y}}\right)\right),
$$

where the parameter $\varphi$ was 0.2 .

\section{Discretisation techniques}

Spatial discretisation of transport eqs. (7), (8) and (9) followed the way of central-symmetric discretisation on a staggered grid (Harlow and Welsh, 1965).

Time integration was independent from spatial discretization. For the energy eq. (9), the second-order accurate explicit Adam-Bashford scheme was implemented:

$$
\begin{gathered}
\frac{\bar{T}^{n+1}-\bar{T}^{n}}{\Delta t^{n}}=R H S^{n+1 / 2}, \\
R H S^{n+1 / 2}=R H S^{n}+0.5 \Delta t^{n} \frac{\left(R H S^{n}-R H S^{n-1}\right)}{\Delta t^{n-1}} .
\end{gathered}
$$

In the case of the momentum eq. (8), a combination of the Adam-Bashford scheme and the projection method was used.

The elliptic pressure equation, which arises from combining eqs. (7) and (8) to satisfy the mass conservation principle, was solved with the Conjugate Gradient method (Ferziger and Perić, 1996).

\section{Stability}

Timestep stability restrictions for time integration were taken from explicit central symmetric scheme for convectiondiffusion equation. The diffusion stability condition implemented in the numerical scheme is formulated as:

$$
\Delta t\left(v+v_{s g d}\right)_{j, i}\left(\frac{1}{\Delta x_{i}}+\frac{1}{\Delta y_{j}}\right)=0.25 .
$$

and the combined convection-diffusion stability condition as:

$$
\Delta t\left(\frac{v_{x}^{2}+v_{y}^{2}}{v+v_{s g d}}\right)_{j, i}=2.0
$$

The largest time step, which satisfied both conditions, eqs. (16) and (17), was used for time marching.

\section{RESULTS}

Simulations were performed for Rayleigh numbers $10^{6}$, $10^{7}, 10^{8}, 10^{9}, 10^{11}$ and $10^{13}$. The Prandtl number was 0.8 in all cases. Numerically calculated Nusselt numbers were averaged along the boundaries and through simulated time. Averaged Nusselt numbers were than compared with experimentally obtained results of Nourgaliev et al. (1997), and Steinberner and Reineke (1978). The comparison was presented in Horvat et al. (1998). Observable differences between experimental and numerical results first occur at Rayleigh number $10^{8}$, when the fluid circulation becomes unsteady. This makes the heat transfer difficult to assess. It was shown that the probability densities of Nusselt number distributions are also important. Namely, to realistically determine the threat to lower plenum integrity, the 
probability of thermal loadings has to be known. The non-linear behavior of the fluid at high Rayleigh numbers produces probability density functions different from Gauss functions. As a consequence, the most probable values of heat transfer do not coincide with time-averaged values (Horvat et al., 1999).

In the present paper, the mechanisms of unsteady momentum and heat transfer will be explained. The time minimum (eq.19), average (eq.18) and maximum (eq.20) local Nusselt numbers along the boundaries were calculated after the thermal steady state was reached.

$$
\begin{aligned}
& N u\left(x_{i}\right)_{\text {time aver }}=\frac{1}{t} \int_{t} N u\left(t, x_{i}\right) d t, \\
& N u\left(x_{i}\right)_{\text {time min }}=\min \left[N u\left(t, x_{i}\right)\right], \\
& N u\left(x_{i}\right)_{\text {time max }}=\max \left[N u\left(t, x_{i}\right)\right]
\end{aligned}
$$

The thermal steady state was defined as a balance between thermal energy production and losses through boundaries of the simulation domain. The time period during which the time average Nusselt number was calculated was $1 / 2$ of the simulation time.

\section{Laminar fluid motion}

At Rayleigh numbers $10^{6}$ and $10^{7}$ the fluid circulation is stable, forming two counter-rotating vortices (fig. 1).

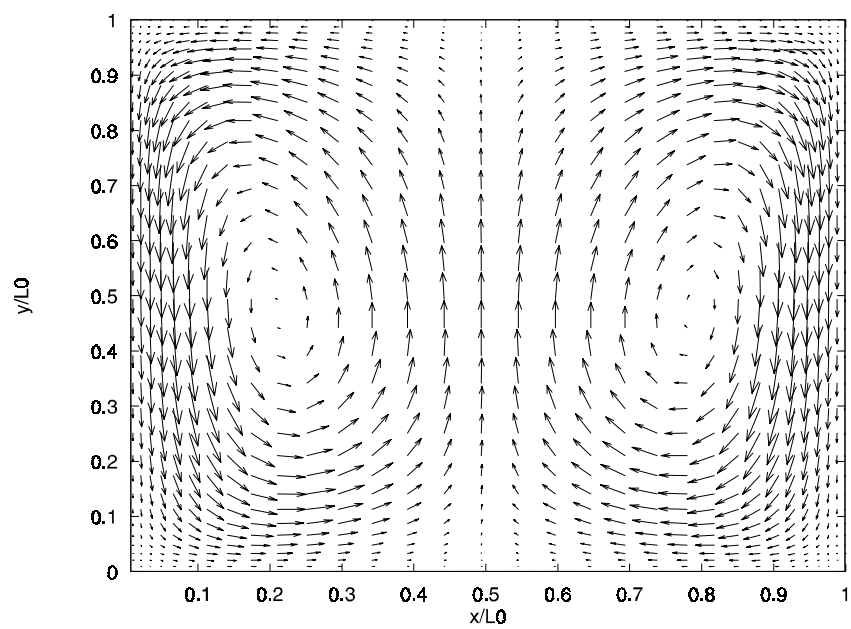

Figure 1: Fluid circulation at $\mathrm{Ra}=10^{6}, \mathrm{Pr}=0.8$.

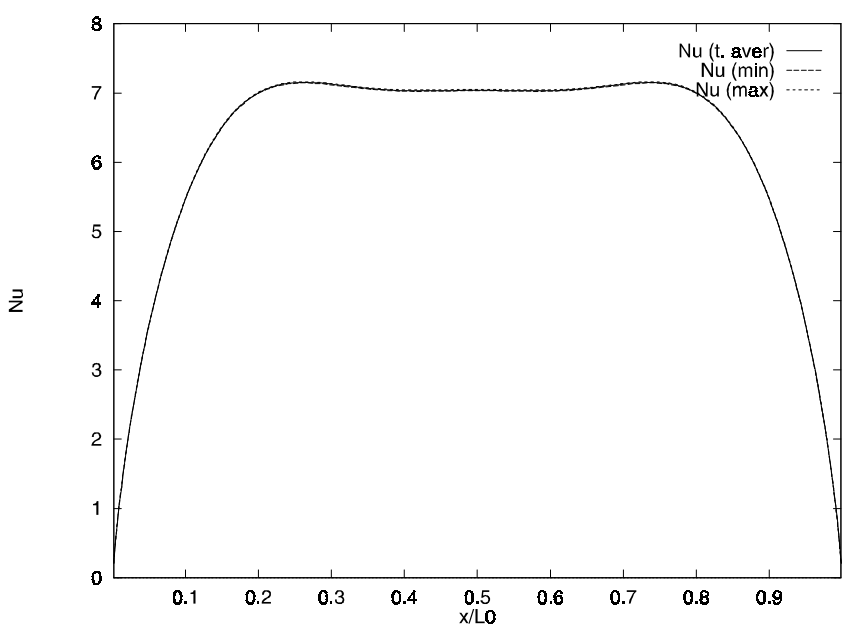

Figure 2: Nusselt number at the bottom boundary, $\mathrm{Ra}=10^{6}, \operatorname{Pr}=0.8$

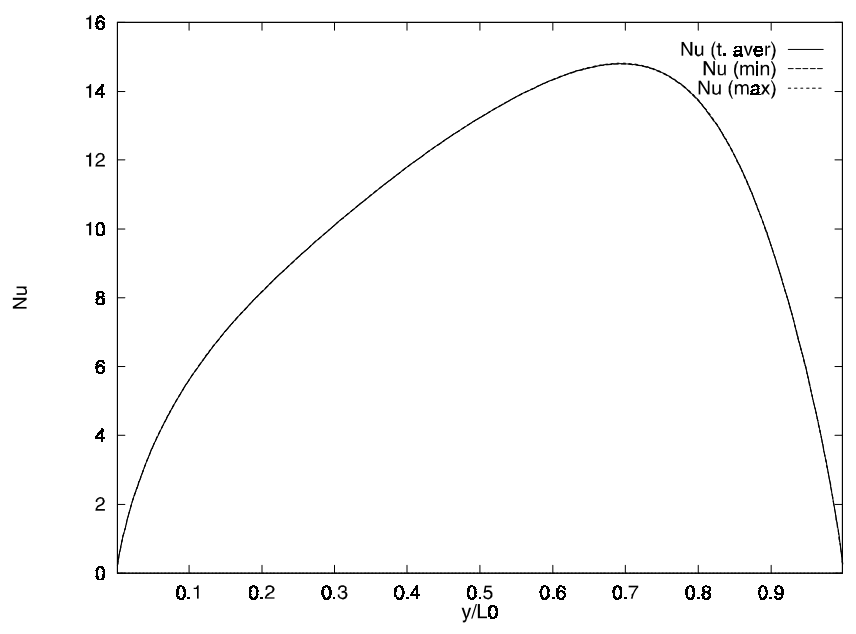

Figure 3: Nusselt number at the side boundary, $\mathrm{Ra}=10^{6}, \mathrm{Pr}=0.8$

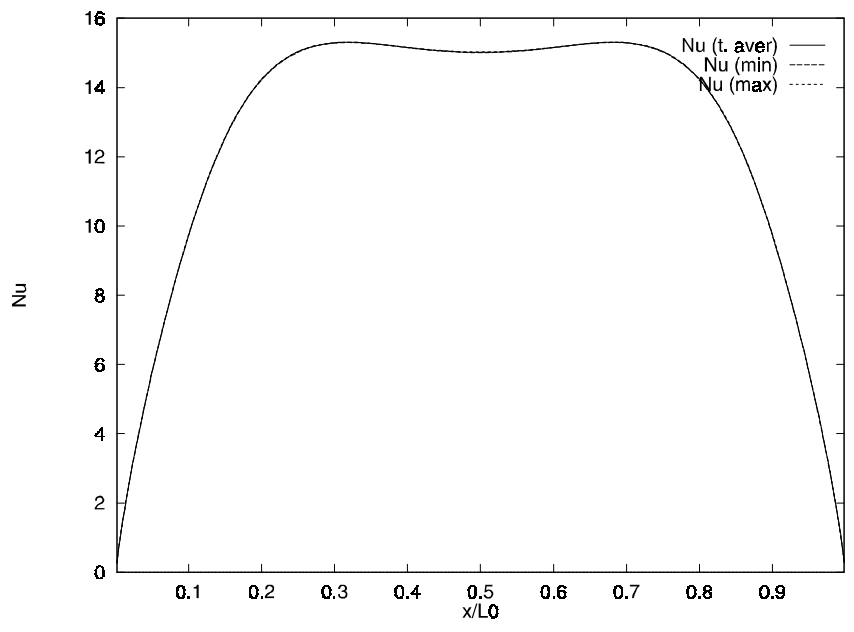

Figure 4: Nusselt number at the upper boundary, $\operatorname{Ra}=10^{6}, \operatorname{Pr}=0.8$ 
On figs. 2-4 the minimum, time-average and maximum Nusselt numbers for different boundaries are presented. Because the system reaches a steady state, the minimum, timeaverage and maximum Nusselt numbers coincide at the end of the simulation. In the case of Rayleigh number $10^{7}$, a steady state was also reached.

Generally, the Nusselt numbers are low at the bottom (fig. 2) and increase towards the upper boundary of the simulation domain (fig. 3). A local maximum is reached in the upper corners on the sidewalls. Similar values of Nusselt numbers may also be observed on parts of the upper boundary (fig. 4).

At Rayleigh number $10^{7}$, Rayleigh-Taylor instabilities, which are the result of intensive cooling of the fluid at the top, cause a "jet" of cold fluid from the upper boundary towards the center of the simulation domain to appear. The jet produces two additional and stable vortices in the middle of the upper boundary. The consequence of the described process is a deep valley in the Nusselt number curve on the upper boundary.

\section{Bifurcation behavior}

At Rayleigh number $10^{8}$, the fluid circulation does not reach a steady state after the initial thermal transient. The system experiences a bifurcation behavior, where it oscillates between two extreme modes (fig. 5 and 6). This results in timedependence of the Nusselt number, where the minimum, timeaverage and maximum Nusselt number can be distinguished as shown on figs. 7, 8 and 9. The differences between minimum, time-average and maximum local Nusselt numbers are larger on the side and upper boundaries where the Nusselt numbers are also higher than on the bottom boundary.

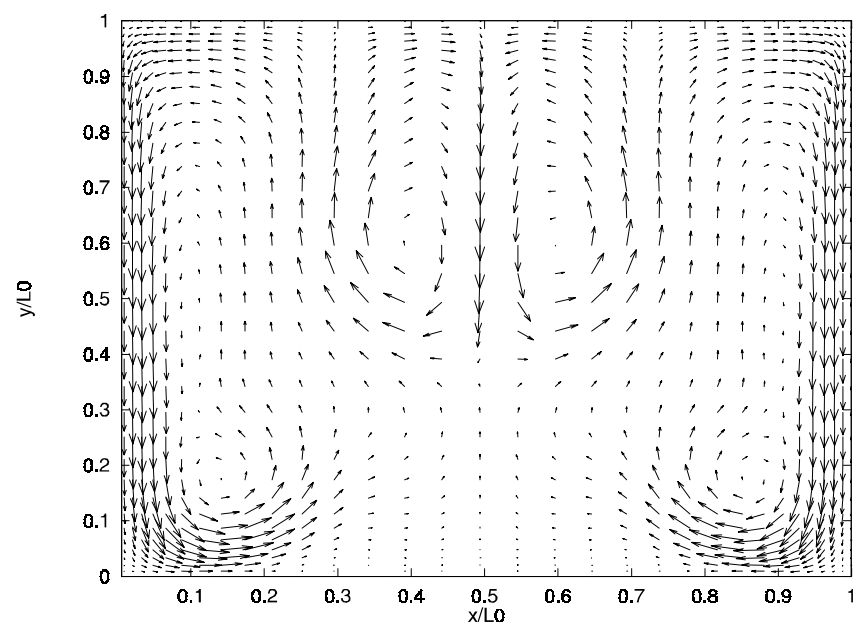

Figure 5: Fluid circulation at $\operatorname{Ra}=10^{8}, \operatorname{Pr}=0.8$

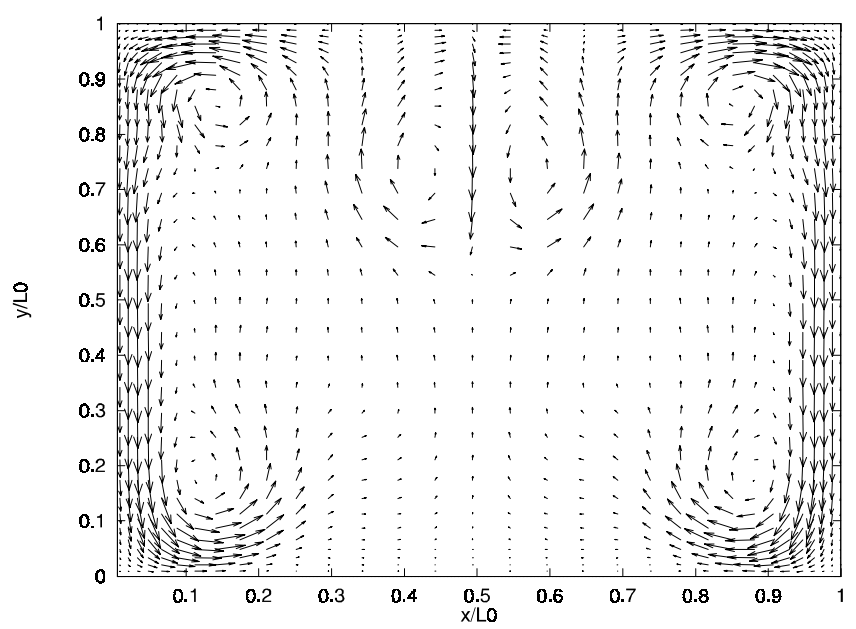

Figure 6: Fluid circulation at $\mathrm{Ra}=10^{8}, \mathrm{Pr}=0.8$

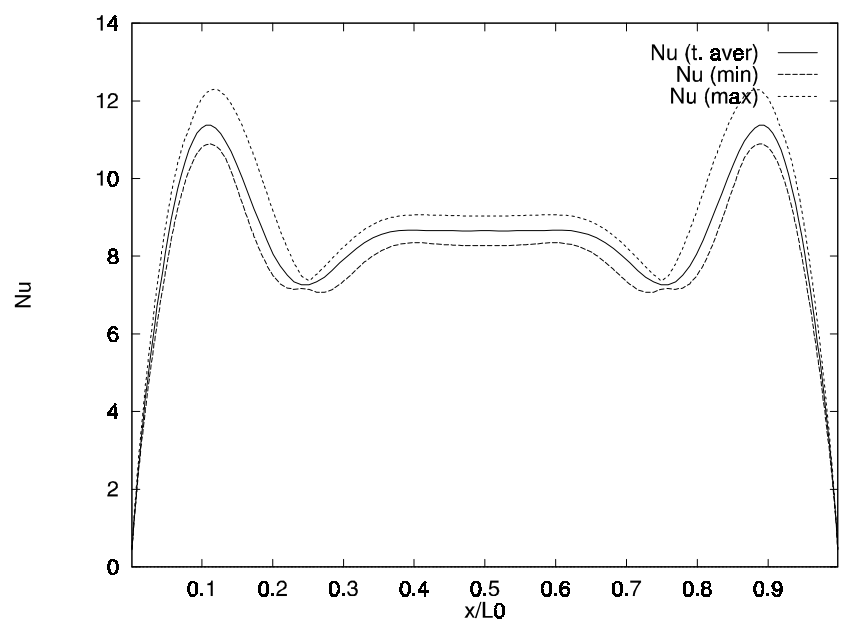

Figure 7: Nusselt number at the bottom boundary, $\operatorname{Ra}=10^{8}, \operatorname{Pr}=0.8$

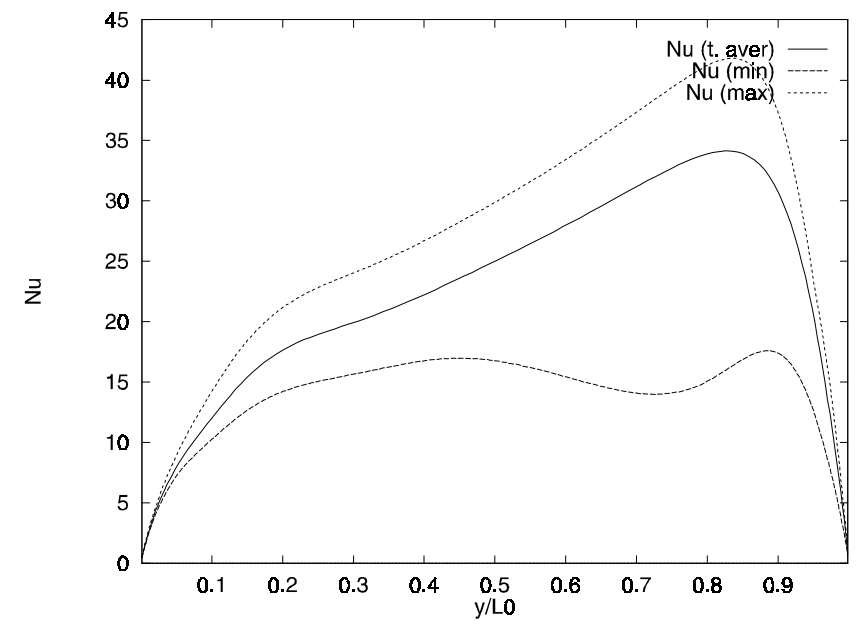

Figure 8: Nusselt number at the side boundary, $\operatorname{Ra}=10^{8}, \operatorname{Pr}=0.8$ 


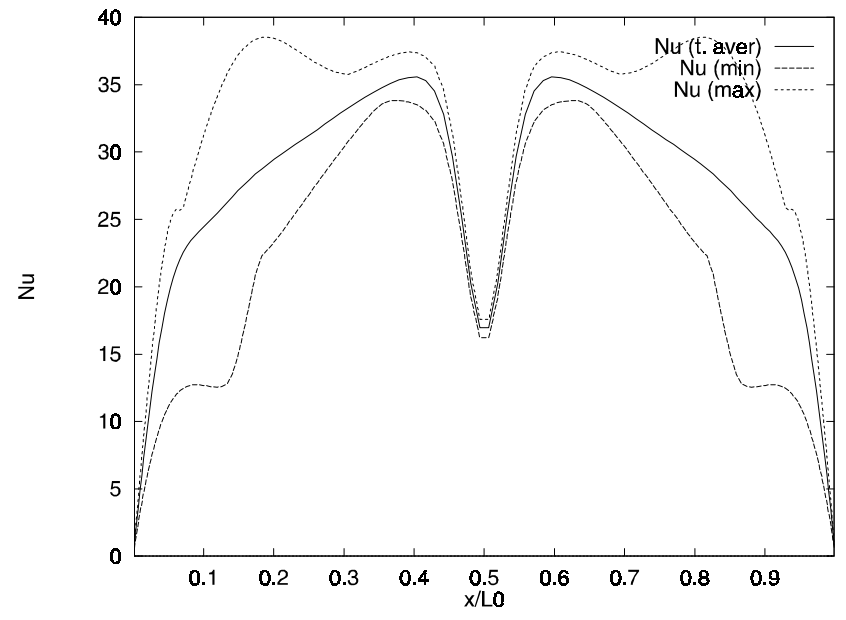

Figure 9: Nusselt number at the upper boundary, $\operatorname{Ra}=10^{8}, \operatorname{Pr}=0.8$

The two local peaks on the bottom Nusselt number curve (fig. 7) reveal the additional vortices caused by downward flow at the sidewalls. The phenomenon is not limited only to the rectangular geometry but can be also observed in hemispherical and elliptical geometries as presented by Nourgaliev et al. (1997).

In the middle of the upper boundary (fig. 9) the Nusselt number inclination reveals a persistent jet of cold fluid towards the center of the simulation domain, which changes its strength in time (fig. 6). On both sides the large differences between minimum, time-average and maximum Nusselt number show additional locations where Rayleigh-Taylor instabilities occur, which results in the appearance of time-dependent vortices (fig. 6).

The higher Rayleigh number causes additional fluid structures to appear. During the heat-up transient or when the Rayleigh number is modest $\left(\mathrm{Ra}=10^{6} \ldots 10^{8}\right)$ the symmetry is preserved and the vortices always appear in pairs. This can be clearly observed on figs. 1, 5, 6 and 13 .

\section{Instabilities and transition to turbulence}

When the Rayleigh number is further increased $\left(\mathrm{Ra}>10^{8}\right)$, the fluid motion becomes asymmetric due to time-dependent vortices. Because of instabilities, which appear at the boundaries of the simulation domain, first local and then global transition from laminar to turbulent motion appears. This can be identified as a sharp scale reduction of fluid motion.

Vortex formation and strong mixing of cold and hot fluid can be first observed in the lower corners of the simulation domain (as on figs. 5 and 6). Namely, the liquid is flowing downwards in a very narrow "boundary layer". Because of intensive cooling through the isothermal side wall, the velocity of the flow is increased. In the lower region of the simulation domain, when the accelerating downward flow meets slow buoyancy flow, strong mixing occurs. The vortices, which can be distinguished from the temperature field (fig. 10), first appear symmetrically in both corners of the simulation domain. When the Rayleigh number is increased to $10^{11}$, the whole lower region becomes unsteady with no permanent fluid structures (fig. 11).

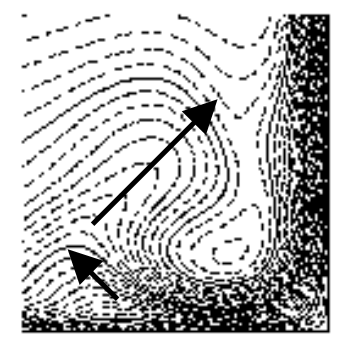

\section{Figure 10: Temperature field in lower corners of simulation domain, $\mathrm{Ra}=10^{8}, \mathrm{Pr}=0.8$ (arrows indicate flow direction)}

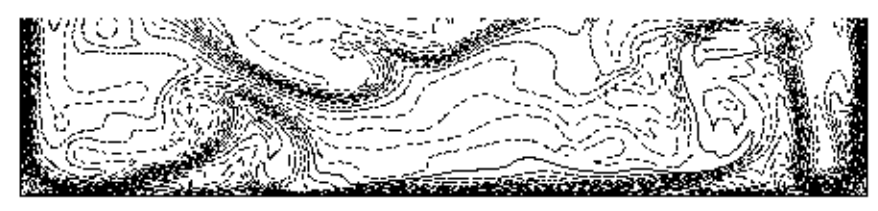

\section{Figure 11: Unsteady temperature field at lower boundary, $\operatorname{Ra}=10^{11}, \operatorname{Pr}=0.8$}

At Rayleigh number $10^{9}$ or higher, the counterflows at the side boundaries also cause Kelvin-Helmholz instabilities. When the perturbation in the boundary layer flow is large enough, it intensifies, producing large separated waves. As the temperature contour field on fig. 12 demonstrates, the temperature of the wave core is lower than the surrounding temperature. This makes wave growth self-bounded and unable to globally influence the general circulation pattern.

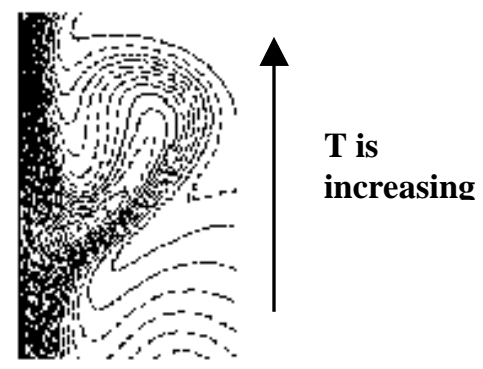

Figure 12: Kelvin-Helmholz instability at side boundary, $\operatorname{Ra}=10^{9}, \operatorname{Pr}=0.8$ 
A phenomenon, which influences the fluid circulation more fundamentally, is the Rayleigh-Taylor instability. The upper boundary of the simulation domain is isothermal with the temperature lower than the fluid temperature. The rising fluid is cooled on the upper boundary and the density is increased. The described situation leads to Rayleigh-Taylor instabilities already at relatively low Rayleigh numbers $\left(\mathrm{Ra}=10^{7}\right)$ and to jet formation. Simulations also revealed the symmetry of jet appearance during heat-up period (fig. 13) or at Rayleigh numbers lower than $10^{9}$.

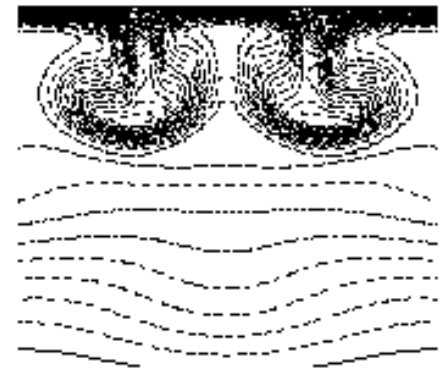

Figure 13: Symmetric Rayleigh-Taylor instability at upper boundary, $\operatorname{Ra}=10^{11}$, $\operatorname{Pr}=0.8$

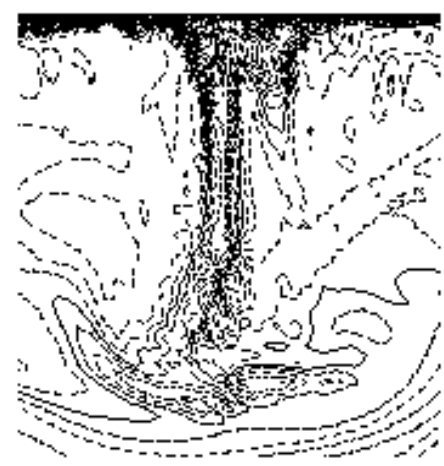

Figure 14: Development of Rayleigh-Taylor instability at upper boundary, $\mathrm{Ra}=10^{13}, \mathrm{Pr}=0.8$

At higher Rayleigh numbers the symmetry disappears. The jets become stronger, disrupting the thermally stratified flow in the core of the simulation domain. The position of appearance and the shape of jets become random (fig. 14).

The described behavior of fluid motion strongly influences heat transfer conditions on the boundaries of the simulation domain. As presented on figs. 15-17 the Nusselt number curves lose the shape which was characteristic in laminar (figs. 2-4) or bifurcation flow regime (figs. 7-9). The shape depends mostly on local fluid structures. Local peaks in Nusselt number function indicate a modest size of fluid structures, which is characteristic for turbulent flow regime. The differences between minimum, time-average and maximum local Nusselt numbers increase with increasing Rayleigh number.

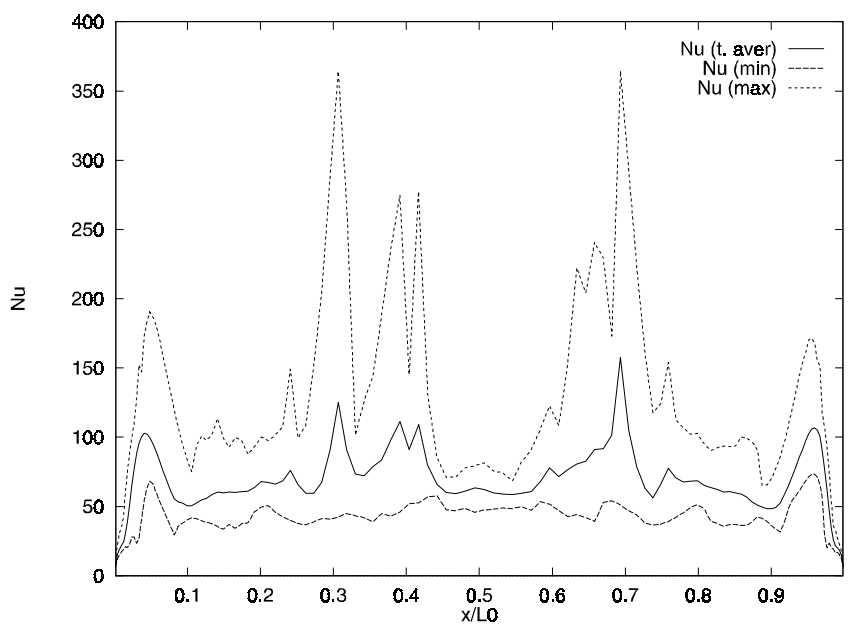

Figure 15: Nusselt number at the bottom boundary, $\mathrm{Ra}=10^{13}, \operatorname{Pr}=0.8$

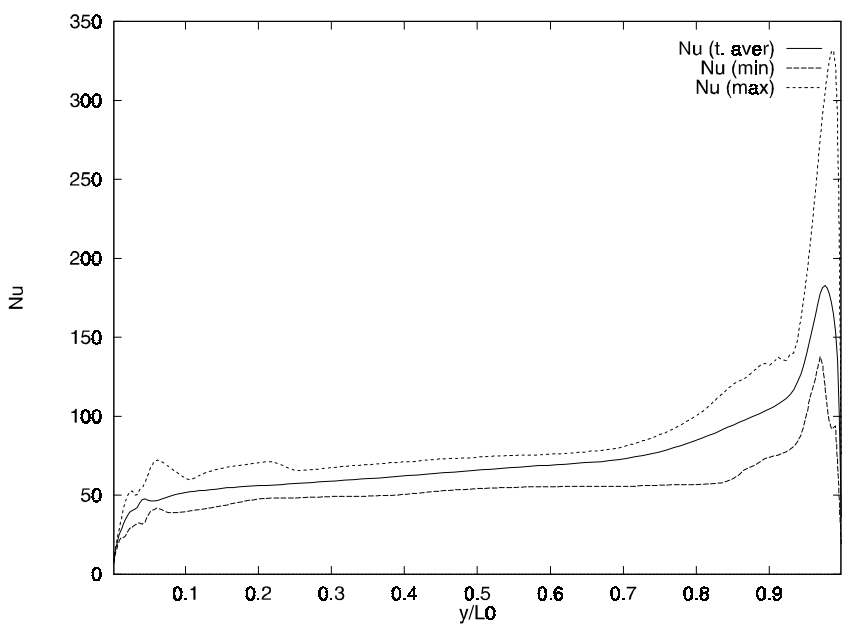

Figure 16: Nusselt number at the side boundary, $\mathrm{Ra}=10^{13}, \operatorname{Pr}=0.8$

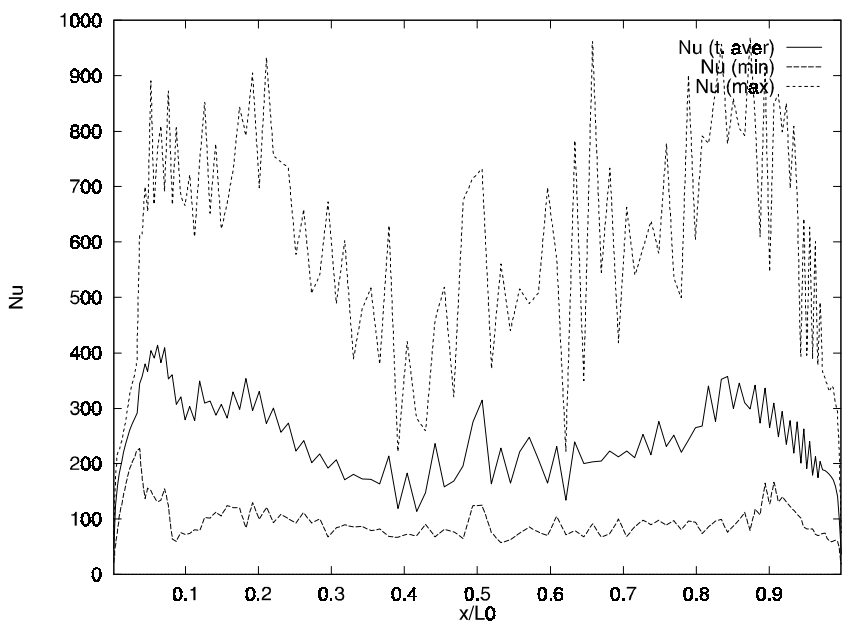

Figure 17: Nusselt number at the upper boundary, $\mathrm{Ra}=10^{13}, \operatorname{Pr}=0.8$ 
The weak similarity between minimum, average and maximum Nusselt number functions also confirms the timedependence of fluid structures and the unsteadiness of the heat transfer process on the bottom boundary.

The described structure dependence of fluid motion at the boundaries of the simulation domain makes the time average of heat transfer hard to assess. To overcome this difficulty the time-average values should be supplemented with probability distributions of related variables.

\section{CONCLUSIONS}

Two-dimensional numerical simulations of a fluid flow with internal heat generation in a rectangular cavity at Rayleigh numbers from $10^{6}$ to $10^{13}$ were performed to examine the Nusselt number behavior on the boundaries of the simulation domain. The dynamics of Nusselt number was also analyzed to identify the mechanisms responsible for transition from laminar to turbulent fluid motion. To capture the fluid subgrid motion, a Large-Eddy Simulation (LES) Smagorinsky model was applied.

The simulations disclosed that steady-state heat transfer can be achieved till $\mathrm{Ra}=10^{7}$. At $\mathrm{Ra}=10^{8}$ the first instabilities are observed which results in bifurcation behavior of the system. When the Rayleigh number is further increased, the fluid flow becomes unsteady with no permanent fluid structures.

The results reveal large intervals between the lowest and the highest calculated Nusselt numbers on the boundaries of the simulation domain. The intervals also increase asymmetrically with increasing Rayleigh number as a consequence of instability and turbulence appearance. This suggests that in the future, the single-value calculations of heat transfer values in the lower plenum should be supplemented with probability distributions.

\section{REFERENCES}

Asfia, F.J., Dhir, V.K., 1996, "An experimental study of natural convection in a volumetrically heated spherical pool bounded on top with a rigid wall", Nucl. Eng. Design, 163, pp. 333-348.

Decker, W.J., 1996, "Numerical studies of bifurcation and chaos in natural convection", Ph.D. Thesis, Virginia University.

Dinh, T.N., Nourgaliev, R.R., 1997, "Turbulence modeling for large volumetrically heated liquid pools", Nucl. Eng. Design, 169, pp. 131-151.

Eidson, T.M., 1985, "Numerical simulation of the turbulent Rayleigh-Benard problem using subgrid modeling", J. Fluid Mech., 158, pp. 245-268.

Ferziger, J.H., Perić, M., 1996, "Computational methods for fluid mechanics", Springer Verlag, Berlin, pp. 101-106.

Harlow, F.H., Welch, J.E., 1965, "Numerical calculation of time-dependent viscous incompressible flow of fluid with free surface", Phys. of Fluids, 8, pp. 2182-2189.
Hindmarsh, A.C., Gresho, P.M., Griffiths, D.F., 1984, "The stability of explicit Euler time-integration for certain finite difference approximations of the multi-dimensional advectiondiffusion equation", Int. J. Num. Meth. Fluids, 4, pp. 853-897.

Horvat, A., 1998, "Modeling of natural convection phenomena in nuclear reactor core melt", M.Sc. Thesis, Faculty of Mathematics and Physics, University of Ljubljana, http://www2.ijs.si/ ahorvat.

Horvat, A., Kljenak, I., Marn, J., 1998, "Numerical Estimation of Reactor Core Melting", Transactions of ANS, Vol. 79, pp. 380-381.

Horvat, A., Kljenak, I., Marn, J., 1999, "Dynamic behavior of the melt pool at severe accident conditions", to be presented at 9th Int. Topical Meeting on Nuclear Reactor Thermal Hydraulics (NURETH-9), San Francisco, California.

Kulacki, F.A., Goldstein, R.J., 1972, "Thermal convection in a horizontal fluid layer with uniform volumetric energy sources", J. Fluid Mech., 83, pp. 375-395.

Nourgaliev, R.R., Dinh, T. N., Sehgal, B.R., 1997, "Effect of fluid Prandtl number on heat transfer characteristics in internally heated liquid pools with Rayleigh number up to $10^{12 "}$, Nucl. Eng. Design, 169, pp. 165-184.

Steinberner, U., Reineke, H.H., 1978, "Turbulent buoyancy convection heat transfer with internal heat sources", Proceedings of 6th Int. Heat Transfer Conference, Vol. II, Toronto, Canada, pp. 305-310.

Theofanous, T.G., Liu, C., Additon, S., Angelini, S., Kymäläinen, O., Salmassi, T., 1997, "In-vessel coolability and retention of a core melt", Nucl. Eng. Design, 169, pp. 1-49. 